\title{
Separated Flow Topology in Compressors
}

\author{
James V. Taylor \\ Whittle Laboratory, University of Cambridge, United Kingdom \\ Email: jvt24@cam.ac.uk
}

When a multi-stage high-speed compressor is operated away from its design point extreme incidence is caused in some blade rows. This results in large, localised separations which are three-dimensional in nature. In this paper topological reasoning is used to describe the behaviour of these three-dimensional separations. It is shown that two classes of separation exist: One in which the flow progresses from attached to separated in a smooth way; and another where there is a discontinuity in the response of the flow topology.

It is shown that the global structure of the flow depends upon the type of topological response that occurs. When the response is discontinuous, non-axisymmetric cells of separated blades are formed. When the response is smooth, the resultant separated flow is axisymmetric. The paper is split into two broad sections: The first section presents examples of the two different classes of topological response that can occur in a single blade row, it also shows how an engineer can achieve a different response by altering the blade design. The second section covers the analysis of a multi-stage high-speed compressor. The compressor initially presents discontinuous behaviour with rotating cells of separations. It is then redesigned to reduce the severity of the cell behaviour or remove it entirely.

\section{Introduction}

When a multi-stage high-speed compressor is operated away from its design point it will mismatch and large separations will be present, these separations are $3 \mathrm{D}$ in nature and most often occur close to the endwalls. The separated blades can group together into cells, and the cells rotate around the annulus. This paper will argue that the formation of cells is linked to the topology of the flow as it switches from attached to separated. It is shown that an engineer can control the topology of the separation by changing the design of the blades. With redesign it is possible to develop a compressor which does not form cells when it is operated away from its design point. It is possible to get all of the blades to separate axisymmetrically.

Designing compressor blade rows that don't exhibit cell behaviour when they separate would have two clear benefits: It would remove the unsteady loading caused as cells rotate around the annulus, preventing forcing of the blades at frequencies close to their natural modes. It also reduces distortion onto neighbouring rows upstream and downstream and can extend the operating range of the whole machine.

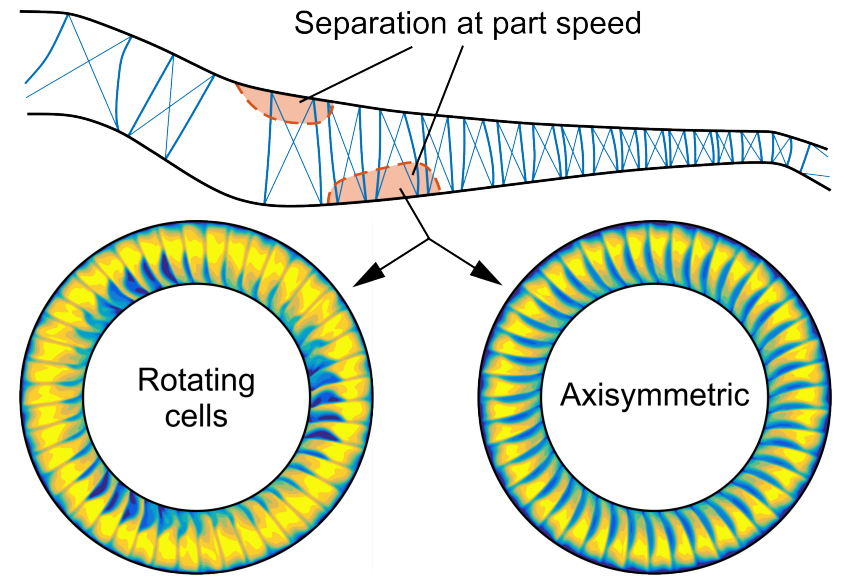

Fig. 1. CONTOURS OF ENTROPY FUNCTION DOWNSTREAM OF STATOR 2 SHOWING THE EFFECT OF SEPARATION CLASS ON OFF-DESIGN BEHAVIOUR

In this paper an 8 stage axial compressor is used as an example. Figure 1 shows the axial compressor studied by Dodds and Vahdati [1] \& [2]. When this compressor is running at part speed 3D separations occur either in rotor 1 (R1) near the casing or in stator 1 (S1) and rotor 2 (R2) near the hub. Two cases are run in 3D CFD: First, the datum geometry as reported previously. When this case is run at $85 \%$ speed open corner separations form in S1 and R2 near the hub. These are shown in a cut plane downstream of R2 in the left hand side of Figure 1, the separated blades group into 3 cells that rotate at $30 \%$ of the shaft speed. In the second case the blades have been redesigned with increased sweep and lean in the hub of S1 and R2. At the same operating condition these blades are also separated because of the high incidence. However, the mechanism by which the separation occurs has changed; the blades now fail by trailing edge separation. The effect of 3D design on separation mechanisms is discussed in detail by Taylor \& Miller [3]. In this redesigned case the separated blades do not group together into rotating cells and the flow in the annulus is completely axisymmetric.

In order to design a compressor to operate successfully at an operating condition other than its design point the topological changes the flow undergoes as it separates should be investigated and understood. The aim of this paper is to present a framework by which a designer can apply this philosophy to their machines. It is split into four sections: 
First, a definition of topological discontinuity. How the topology of the flow in a compressor blade row responds with incidence. The difference between a smooth topological response and a discontinuous response.

Second, methods for best practice computation of separated flow and stages operating beyond their maximum pressure rise.

Third, examples of the different types of flow separation that occur in 2D and in 3D. The development of the topology as flow separates is investigated in detail in order to help identify behaviours in more complex applications such as those in multi-stage machines.

Fourth, behavioural analysis of the example 8 stage axial compressor as it is operated at part speed. Design modifications are made to problematic blade rows, the improvements to the flow are investigated and specific recommendations to designers are made.

\section{Discontinuities in Topology}

When incident flow angle onto a row of compressor blades is increased the topology of the flow will respond in one of two ways. Either, separation will occur progressively and the topology changes smoothly between both states. Or, a critical incidence will exist where the topology can jump from one branch of stable solutions to another; this is a discontinuous topological response to incidence.

Figure 2 shows examples of these two kinds of responses occurring on the hub end of a shrouded stator blade. Consider the flow as computed at low incidence in case A, this is a single passage and steady calculation. Therefore, the flow that is converged must be an axisymmetric and equilibrium solution to the RANS equations that the solver is modelling. As incidence is increased from A to B, the topology changes as the size of the closed corner separation grows and the separation line moves further up the span. Between these two points there are no sudden changes in the structure of the flow, it merely shifts and rescales to cope

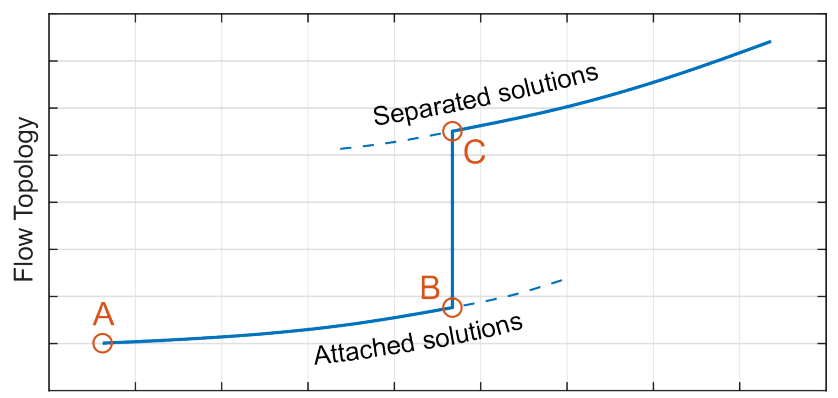

Incidence
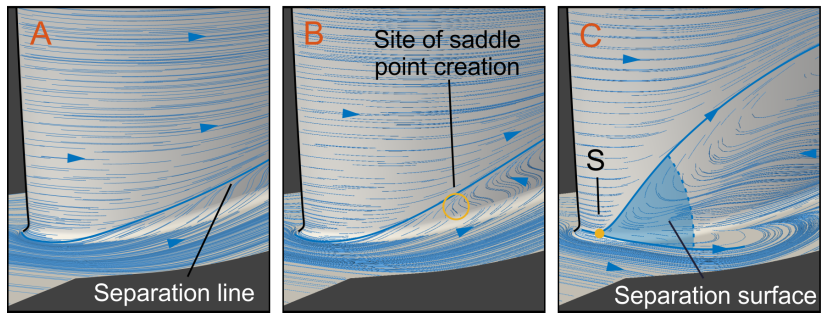

Fig. 2. TOPOLOGICAL RESPONSE TO INCIDENCE INCREASE with the new operating condition. The transition between these two axisymmetric equilibrium solutions is topologically smooth. No sudden changes in loss generation or turning are seen either.

However, if the incidence is increased further a discontinuity is encountered. The corner separation switches from a closed topology to an open topology [3]. In case B, when the corner separation is closed, it is marked only by a single separation line where the endwall boundary layer meets the blade boundary layer. In case $\mathrm{C}$, when the corner separation is open, it has two separation lines. One on the blade as before and a second one now on the hub endwall. At the root of these two separation lines is a saddle point which splits the flow.

A critical incidence exists where this saddle point is formed and the solution of the flow jumps from one branch to another. No possible axisymmetric equilibrium solution exists between these two branches. In a real machine, with many blades in a row, the flow in different parts of the annulus is able to occupy states on both branches. Cells of separated blades rotating around the annulus would be observed as the solutions switch from one branch to the other and back. In this paper it will be shown that cell behaviour can only be observed when discontinuities in topology are present.

\section{Methods}

Three different computational models are used in this paper: First, 2D NACA 6-series aerofoils of different thicknesses are used in an idealised 2D compressor model. The sections are staggered to $20^{\circ}$ and spaced at a pitch-chord of 1.2. The 2D models can be throttled to increase the relative incidence and provoke different classes of separation.

Second, 3D compressor blades are used in a single row configuration to illustrate topological changes in an idealised 3D environment. The blades in these cases are adapted from modern highspeed designs. Changes are made to their thickness, camber, aspect ratio and stacking to provoke different classes of separation.

Third, the rows used in the multi-stage analysis are taken from a modern 8 stage high-speed compressor. Numerical and experimental analysis was also performed on this compressor in [1] and [2]. The configuration of VSVs used in this paper is the same as that referred to as "A" in [1] and [2]. As the phenomena of interest at part speed operation are observed in the front rows of the machine, only the first two stages and IGVs are modelled to reduce computational cost.

The domain of the simulation used in the case of the datum multi-stage geometry is shown in Figure 3. A convergent-

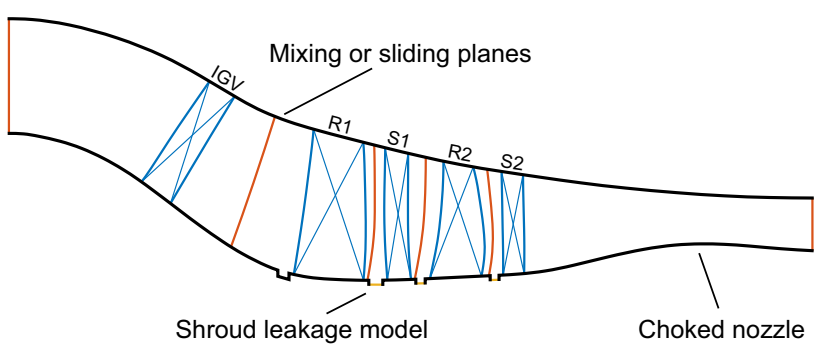

Fig. 3. MERIDIONAL VIEW OF COMPRESSOR DOMAIN 
divergent nozzle is used downstream of S2 to allow solution of the flow beyond the stable operating point of these compressor stages at part speed. In the real machine or a larger calculation the downstream six stages would provide the pressure rise necessary to stabilise these front two stages.

TURBOSTREAM is used to solve all of the flows presented in this paper. It is a 3D structured multi-block code written specifically for turbomachinery, it is described and validated in detail in [4]. The Spalart-Allmaras turbulence model was used with wall functions [5].

Figure 3 shows the position of the mixing planes in steady and sliding planes in unsteady calculations. These have been placed so that the axial platform gaps are always in the same domain as the blade leading edge. This allows the pressure field of the blade leading edge to cause pumping of the flow into and out of the cavity in a realistic manner in a steady calculation. Stub cavities between the platforms were meshed and included in the calculation, net leakage mass flow is determined by a shroud leakage model that links the bottom of the stubs under S1.

For the 3D calculations 250,000 cells are used per blade passage and $y^{+}$is less than 10 on all walls. The meshing process is automated using AUTOGRID. All tip gaps are fully meshed with a two block butterfly topology. Fillets are also meshed with a two block butterfly topology [6] to ensure there is no discontinuity in angle between the fillet and the meridional surface.

Images of the surface limiting streamlines are shown throughout the paper. The images are constructed by randomly seeding points on the surfaces and integrating along the paths formed by the surface skin friction vectors.

For the single row calculations unsteady simulations are run on 10 blade passages and 40 time steps per blade passing are used. In these cases one blade is re-pitched by $1 \%$ to initiate non-axisymmetric behaviour. The multi-stage calculations are run on a $1 / 3$ annulus domain. After converging a frozen rotor case to flush most of the residuals it is possible to converge the unsteady calculation to periodic state in 4 revolutions. 1320 time steps per revolution are used for these calculations.

When blade rows are redesigned with sweep and lean the stacking profiles are in the "true lean" and "true sweep" directions as described in [7]. The quantity of sweep and lean added to the blade rows is described by the angle that the line of blade section centroids makes with the meridional surfaces.

\section{Separated Flow Topology}

The purpose of this section of the paper is to present examples of the separation mechanisms in both 2D and 3D flows. In each example, the topological response is classed to be either smooth or discontinuous.

\section{Two-dimensional Aerofoils}

Separation in 2D flows can occur in one of two ways, either it is initiated at the trailing edge or it is initiated at the leading edge. Previous work on aerofoils used in external flow [8] established that thickness has a strong effect on the type of separation that occurs. When at incidence, thin aerofoils have increased diffusion at the leading edge, while thick aerofoils
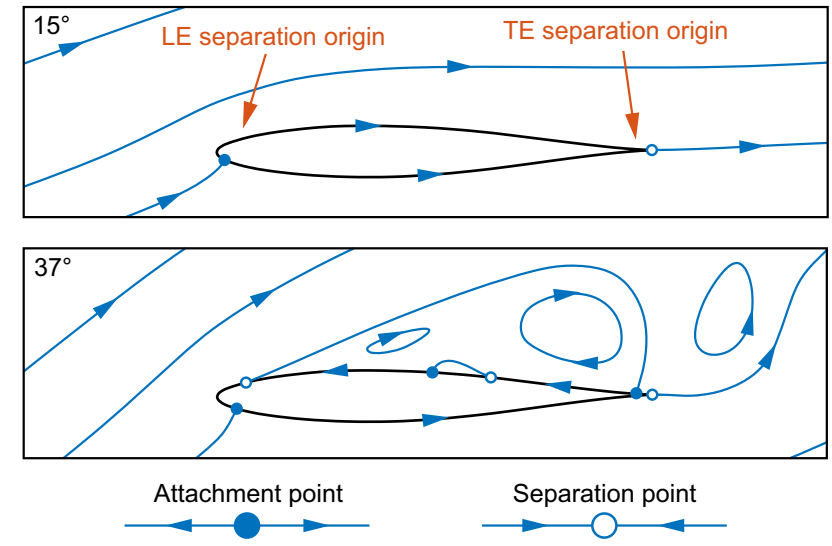

Fig. 4. EQUILIBRIUM TOPOLOGIES OF ATTACHED AND SEPARATED 2D BLADES

have increased diffusion toward the trailing edge.

In this part of the paper the topology of these two separation mechanisms is investigated on two 2D compressor configurations, they are made up of either $6 \%$ or $12 \%$ thickness aerofoils. Symmetric NACA 631 series aerofoils taken from [8] are used with a pitch-chord ratio of 1.2 and a stagger angle of $20^{\circ}$. The $2 \mathrm{D}$ compressor blades are simulated in a CFD calculation and the incidence is increased as the machine is throttled up the characteristic.

When analysing the topology of separations it is helpful to inspect the limiting surface streamlines and the location of their critical points. This approach reduces the number of dimensions to be visualised by one, therefore it is especially useful in 3D flows. The extent of the separated region away from the surface can still be easily inferred from the development of the critical points.

In 2D flows there are only kinds of critical points; the points on the surface where the limiting streamlines change in direction. At attachment points the streamlines run apart and at separation points the streamlines run together. Figure 4 shows schematics of these points. The number of critical points is governed by the index rule shown in Equation 1, derived using the analysis in [9]. This equation holds for all instances in time, thus attachment points and separation points must be formed together in pairs.

$$
\sum \text { Attachments }-\sum \text { Separations }=0
$$

Figure 4 shows two axisymmetric equilibrium topologies as predicted with steady CFD. In the first, at low incidence, the flow is completely attached. This case has one pair of critical points on the surface of the blade, attachment at the leading edge and separation on the sharp trailing edge. In the second case, at high incidence, the suction surface of the blade is fully separated, it has three pairs of critical points at this operating point. The flows around both thin and thick compressor blades at these extremes of operating points are topologically similar. They both have one and three pairs of critical points at the two operating points respectively and the suction surface is completely separated in the high incidence case.

The difference between the two separation mechanisms is the origin location of the second pair of critical points. Figure 5 


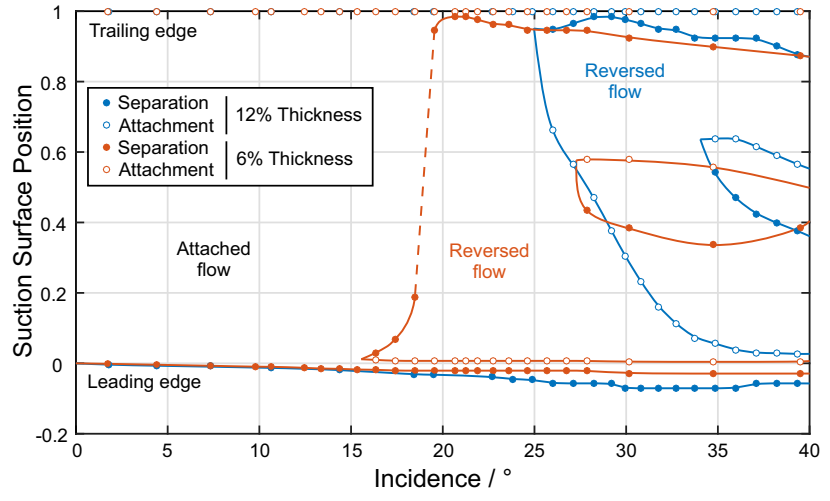

Fig. 5. DEVELOPMENT OF EQUILIBRIUM TOPOLOGY FOR THIN AND THICK 2D BLADES

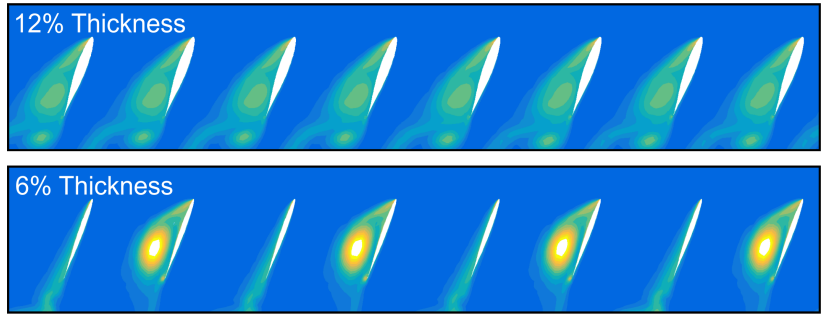

Fig. 6. SNAPSHOT OF UNSTEADY FLOW AT HIGH INCIDENCE FOR THIN AND THICK 2D BLADES

shows the location of the critical points on the suction surface for both sets of compressor blades at a range of incidences. In the case of the $12 \%$ thickness compressor blades at $25^{\circ}$ incidence an attachment and separation point pair is formed close to the trailing edge. As incidence is increased the separation point moves upstream and the separated region increases in size. For this case it is possible to find an equilibrium solution with any size of separation, the topological response of this blade is smooth.

The 6\% thickness compressor blades behave differently. At $16^{\circ}$ incidence a separation bubble opens close to the leading edge but then re-attaches, in [8] this kind of behaviour is referred to as "thin-aerofoil stall". At the critical incidence of $18^{\circ}$ the separation bubble bursts and the attachment point jumps to the trailing edge. It is impossible to find an equilibrium solution with an intermediate size of separation, therefore this blade has a discontinuous topological response to incidence.

To investigate if these separations will occur axisymmetrically or not unsteady RANS is used to compute the flow in a 10 passage domain. Figure 6 shows an instantaneous snapshot of both compressors operating at high incidence. In the $12 \%$ thickness compressor the flow is axisymmetric, all blades are completely separated. In the 6\% thickness compressor the flow alternates between attached and separated. Cells spanning only one blade are formed and rotate in the direction opposite to rotation.

This analysis is repeated for multiple thicknesses between $24 \%$ and $1 \%$. All thicknesses below $8 \%$ were found to exhibit cell behaviour while the thicker blades behaved axisymmetrically. The range of incidences over which cell behaviour was observed is reduced in the case of the thinnest blades. In these cases the magnitude of the topological discontinuity is smaller because the leading edge separation bubble can reattach at larger sizes. Thus when it does detach completely the required change in the topology of the flow is not as large.

From these examples it is concluded that non-axisymmetric cell behaviour is only found in 2D flows when leading edge separations occur.

\section{Three-dimensional Compressor Blades}

Separation in 3D flows on compressor blades can also be split into two groups. Those which are initiated at the trailing edge of the blade and those that are initiated at the leading edge or middle of the blade. Where the separation originates in this second group is primarily driven by the endwall configuration: Midspan leading edge separations can occur on thin high aspect ratio blades. Open corner separations can occur on blades with fixed endwalls, such as rotor hubs and shrouded stators. Tip separations can occur on blades with free endwalls with a gap, such as rotor casings and cantilevered stator hubs.

In this part of the paper the topology of separation is investigated by inspecting the critical points and the limiting streamlines on the surface of the blades. In 3D flows there are three different kinds of critical points, their number is also governed by an index rule as shown in Equation 2. Like in 2D flow this rule requires critical points to be formed in pairs. Saddle points are the most important in separated flow as they make one region of the flow inaccessible to another. A full derivation of critical points and the index rule is given in [9], topology in a compressor application was originally used in [10] and more recently in [3].

$$
\sum(\text { Foci }+ \text { Nodes })-\sum \text { Saddles }=N
$$

Four blades were designed at Mach 0.4 to demonstrate each of the four separation types. Their characteristics as predicted using single passage steady CFD with a choked nozzle are shown in Figure 7. The design modifications made to achieve these different types of separation are summarised in Table 1.

The blades which have trailing edge or midspan leading edge separation are designed with compound sweep and lean to prevent open corner separations on the fixed ends at hub and casing. The case with a trailing edge separation has a thickness

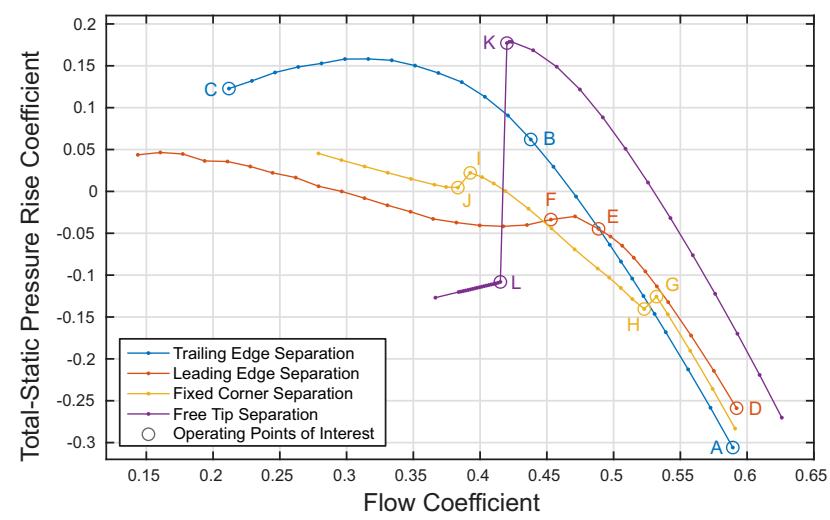

Fig. 7. CHARACTERISTICS OF EXAMPLE 3D BLADES 
Table 1. DESIGN PARAMETERS OF EXAMPLE 3D BLADES

\begin{tabular}{lcccc} 
Separation type & $\begin{array}{c}\text { Leading } \\
\text { Edge }\end{array}$ & $\begin{array}{c}\text { Trailing } \\
\text { Edge }\end{array}$ & $\begin{array}{c}\text { Fixed } \\
\text { Corner }\end{array}$ & $\begin{array}{c}\text { Free } \\
\text { Tip }\end{array}$ \\
\hline Thickness & $1.6 \%$ & $10 \%$ & $3.9 \%$ & $5.8 \%$ \\
Camber style & CDA & Circular & CDA & CDA \\
Aspect ratio & 2.3 & 1.6 & 1.3 & 2.3 \\
Lean or sweep & Yes & Yes & Opposite end & No \\
Endwall type & Shrouded & Shrouded & Shrouded & Tip gap
\end{tabular}
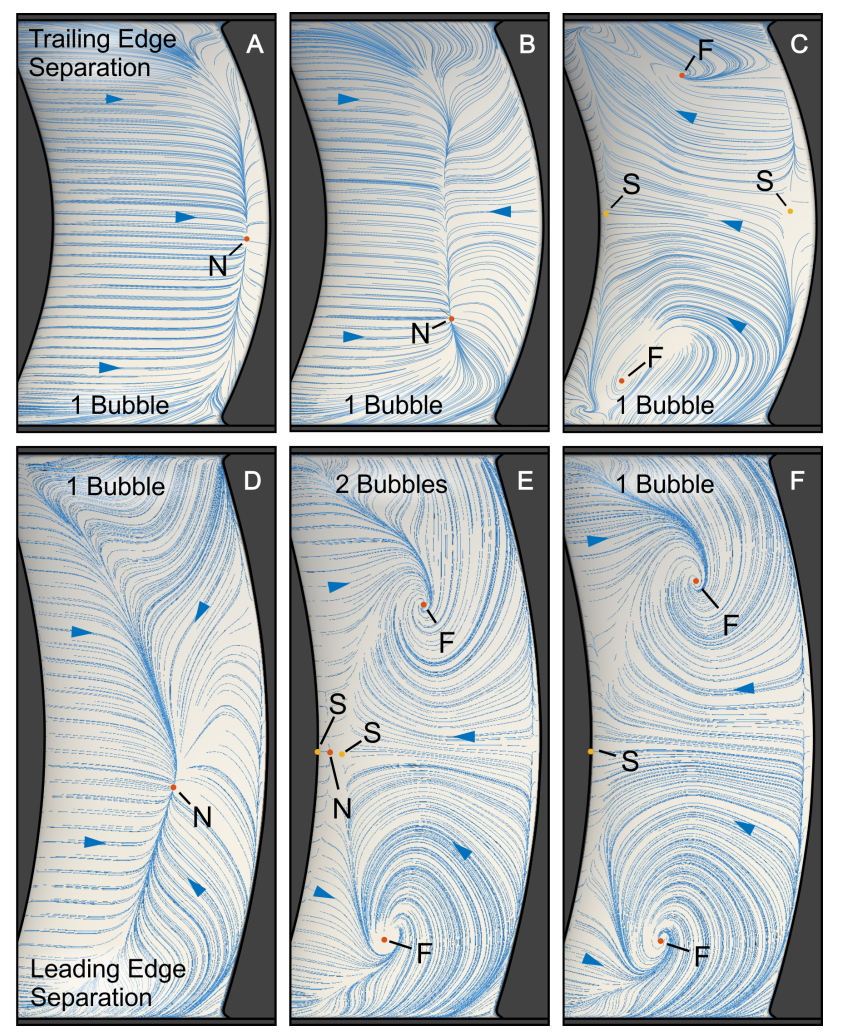

Fig. 8. EQUILIBRIUM TOPOLOGIES OF MIDSPAN SEPARATIONS

at midspan of $10 \%$, a circular arc camber line and an aspect ratio of 1.6. The case with a midspan leading edge separation has a thickness at midspan of $1.7 \%$, controlled diffusion camber line and an aspect ratio of 2.3. All of these design modifications change the balance of diffusion between leading and trailing edge.

Blades which have open corner or free tip separations are designed with sweep and lean to provoke separation at one end only. The case with an open corner separation is designed to represent a stator blade with a shrouded hub, so sweep and lean is used to prevent separation at the casing end. The case with a free tip separation is designed to represent a rotor blade with a tip gap at the casing, no sweep or lean is required at the hub because of the centrifugal effect on the boundary layer.

In the following sections operating points close to the discontinuities in topology are investigated for the four example blades. These are labelled as points A to $\mathrm{L}$ on Figure 7.

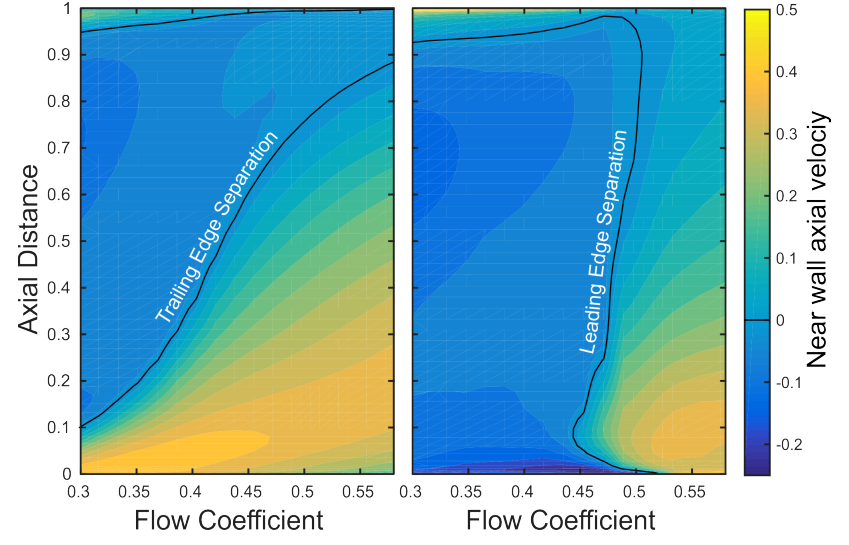

Fig. 9. MIDSPAN NEAR WALL AXIAL VELOCITY

\section{Leading and Trailing Edge Separation}

Figure 8 shows the topology of surface limiting streamlines for the trailing edge and midspan leading edge separations. Over the whole operating range of the trailing edge separation blade the topological response is smooth. At low incidence (A) the flow has a small separation at the trailing edge. As incidence is increased (B to $\mathrm{C}$ ) the separation line moves continually upstream until it reaches the leading edge and the suction surface is fully separated. In the case of the midspan leading edge separation blade there is a discontinuous topological response. At low incidence (D) a separation line sits midway down the chord, this is currently trailing edge separation. As incidence is increased $(\mathrm{E})$ the leading edge separates and re-attaches forming an attachment line upstream of the previously existing separation line. In solution $\mathrm{E}$ the separation line and attachment lines are in close proximity with two saddle points and a node between them. When the incidence is increased past this critical value (F) the first saddle point and the second node coalesce and are destroyed. The separation line jumps to the leading edge and the suction surface is now completely separated.

Figure 9 shows these mechanisms from a different perspective. This figure charts the progress of the separated regions with flow coefficient along the midspan line of the blade. In the left hand plot it can be seen that the trailing edge separation can exist at any size depending on operating point. In the right hand plot the discontinuity in topology occurs for the leading edge separation example blade at a flow coefficient of 0.44 . Here the two bubbles that were in equilibrium with a small region of attached flow at $10 \%$ chord coalesce and burst.

Unsteady RANS was used to simulate the flow in a multipassage domain made up of these two example blade designs. Two instantaneous snapshots of the flow are compared in Figure 10. In the example of the trailing edge separation axisymmetric flow was found over the whole operating range. In the example of the midspan leading edge separation, at an operating point close to the critical flow coefficient of 0.44 , cell behaviour was observed. The cell is confined to the midspan region of the blade only, the endwall regions remain well behaved due to the lean and sweep and thicker sections in this part of the blade. 

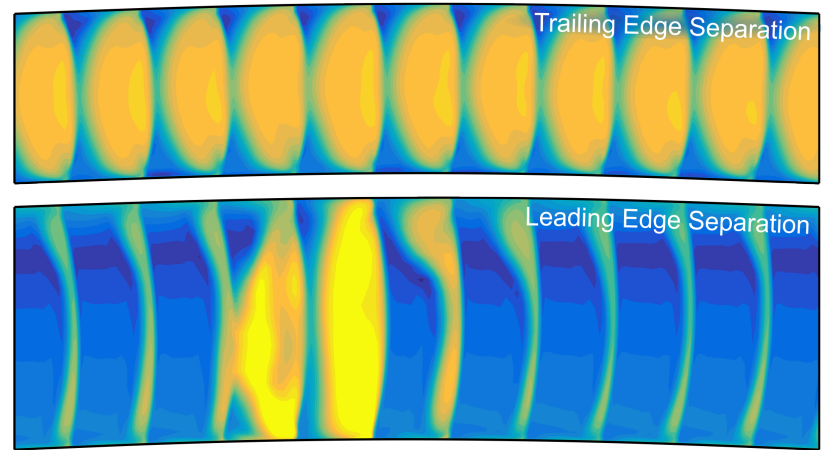

Fig. 10. SNAPSHOT OF CELL BEHAVIOUR IN MIDSPAN SEPARATION BLADES
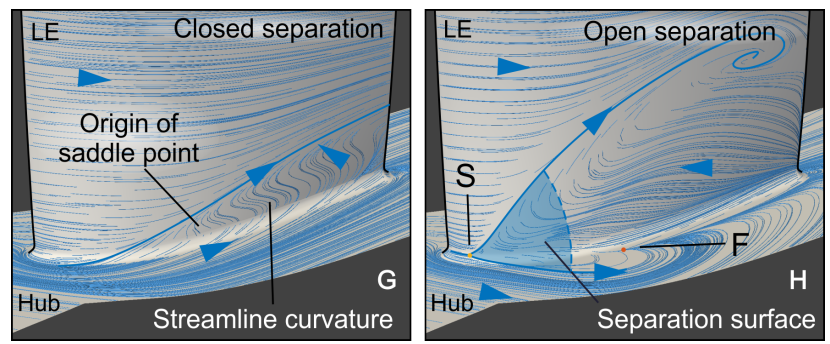

Fig. 11. EQUILIBRIUM TOPOLOGIES OF CLOSED AND OPEN CORNER SEPARATIONS

\section{Fixed Corner Separation}

The example blade which fails by open corner separation has two discontinuities in topology. The first discontinuity is a switch from closed corner separation to open corner separation ( $\mathrm{G}$ and $\mathrm{H})$. Figure 11 shows the equilibrium topology at the two operating points either side of the discontinuity. Below the critical incidence (G) the separation is closed, with only one separation line on the blade surface where the endwall flow meets the blade boundary layer. Above the critical incidence $(\mathrm{H})$ the corner separation is open as it now has two separation lines, the second is on the hub endwall, a separation surface is stretched between the two. At the root of the separation surface is a saddle point which divides the flow into two regions. Taylor \& Miller describe the formation of this saddle point in [3]. Its origin is in the middle of the suction surface where the streamlines have high curvature. In this paper when unsteady simulations are performed close to the opening of the corner separation cell behaviour is found. However, the cells of open corner separations do not rotate around the annulus. Because the blockage caused by the open corner separation is relatively low it is unable to cause the required upstream distortion to trip the neighbouring blade over its critical incidence.

At a further reduced flow coefficient the second discontinuity in topology is encountered (I and J). Figure 12 shows the equilibrium topology either side of the discontinuity. Below the critical incidence (I) the open corner separation has grown in size and the separation line penetrates most of the way up the span. However, the topology is still the same as that shown in Figure 11. Once the incidence is increased beyond its second critical value $(\mathrm{J})$ the topology again jumps to a new branch of solutions. The blade now has a "double open separation". It has two saddle points: one on the fillet near the leading edge as
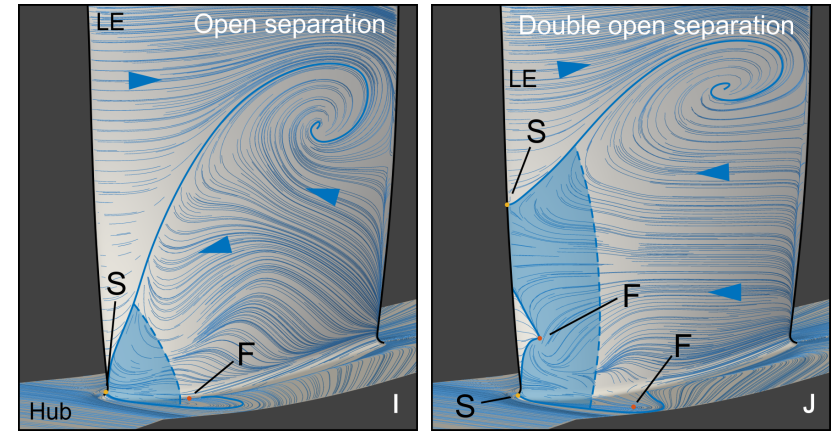

Fig. 12. EQUILIBRIUM TOPOLOGIES OF OPEN AND DOUBLE OPEN CORNER SEPARATIONS
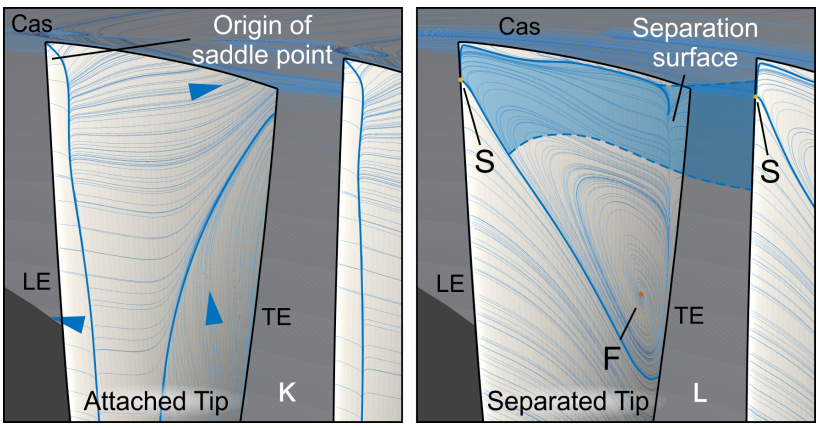

Fig. 13. EQUILIBRIUM TOPOLOGIES OF TIP SEPARATION

before and a new saddle point partway up the span at the leading edge. The size of the separation surface has increased and a larger fraction of the passage is blocked. This kind of separation occurs in the high aspect ratio blading investigated in this paper but does not occur in the low aspect ratio blading presented previously by Taylor and Miller in [3]. Depending on design further discontinuities in topology should be possible with more saddle points being created at the leading edge. Cell behaviour is observed in an unsteady ten passage simulation, in this case large cells covering four passages rotate around the annulus.

\section{Free Tip Separation}

Blades which fail by free tip separation have the largest discontinuity in topology that is described in this section ( $\mathrm{K}$ and L). The equilibrium topologies either side of the discontinuity are shown in Figure 13. Below the critical incidence $(\mathrm{K})$ there is a leading edge separation bubble which reattaches at $10-15 \%$ chord. There is also a separation line caused by a large closed corner separation that has penetrated most of the way up the span from the hub. Above the critical incidence (L) the flow jumps to a different branch of solutions where the tip is separated, in this case the topology has completely changed. A separation line on the suction surface stretches from the leading edge at the tip to midspan at the trailing edge. There is a similar separation line present on the pressure surface and a separation surface is stretched across the whole pitch of the passage. At the root of the separation surface on each leading edge is a saddle point. For the separation surface to exist this saddle point must have been created with its accompanying focus pair in the jump from one branch of solutions to another. 


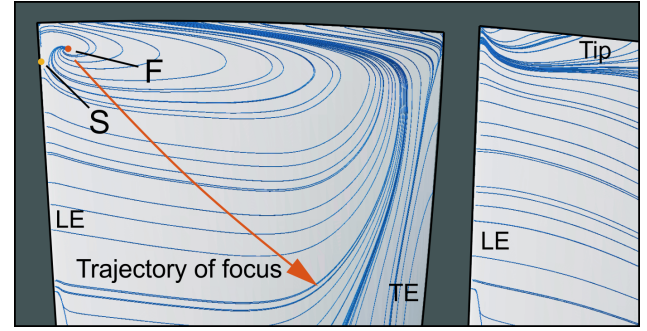

Fig. 14. SNAPSHOT SHOWING CREATION OF SADDLE POINT IN TIP SEPARATION

Figure 14 shows an instantaneous snapshot of an unsteady simulation where a blade is partway through separation. This is a non-equilibrium solution and as time marching continues the flow assumes the fully separated topology in Figure 13. The saddle point and focus pair can only be formed from regions of high streamline curvature, such as the attachment line on the leading edge itself. The saddle point remains at the leading edge and forms the root of the separation surface. The focus moves downstream and down the span as the size of the separation increases. When this unsteady simulation is continued until the solution is periodic in time cells that occupy 4-5 blade pitches are observed.

This part of the paper has shown four examples of separation that are possible in 3D flow in compressor blade rows. The three which have a discontinuous topological response all have a feature in common, the saddle points which form the root of the separations are formed at the leading edge or in the middle of the suction surface of the blade.

\section{Off-design Behaviour of Multi-stage Compressors}

In this section of the paper an example high-speed multi-stage compressor is used to illustrate the effects previously discussed for ideal cases. It comprises of two parts: In the first part the specific separations which occur in the example multi-stage compressor are identified. In the second part 3D redesign is undertaken to improve the cell behaviour of the flow.

\section{Separations at Part Speed}

In a real machine it can be difficult to identify which type of separation is occurring and whether the topological response is smooth or discontinuous. In this part of the paper the topology of the flow in the front two stages of the high-speed example compressor is investigated to illustrate how the techniques can be used.

Work by Dodds et al in [1] and [2] showed that the front stages of this example compressor were susceptible to two modes of separation and cell behaviour. Separation would occur in either the tips of R1 or the hubs of S1 and R2. By changing the VSV schedule and the shaft speed, either mode could be amplified. In this paper the example compressor is considered at two operating points at $85 \%$ speed, these are plotted in Figure 15. Close to the peak pressure rise of the first two stages R1 separates at the tip. When the two stages are run on the working line S1 and R2 separate. Hysteresis is seen in Figure 15 in two locations where the separations change location from $\mathrm{S} 1$ and R2 hub to R1 tip.

Figure 16 shows the equilibrium topology predicted at the

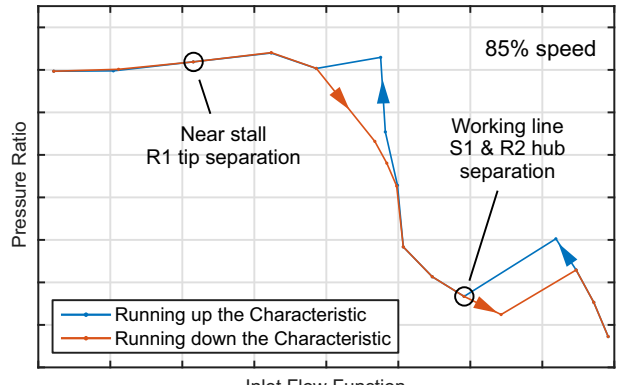

Iet Flow Function

Fig. 15. CHARACTERISTICS AND OPERATING POINTS OF STAGES 1 AND 2

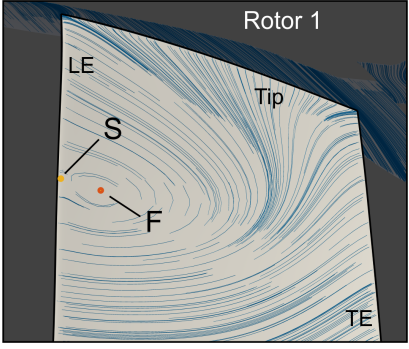

Free tip separation

Fig. 16. EQUILIBRIUM TOPOLOGY OF SEPARATED FLOW ON DATUM R1

top of the characteristic. At this operating point a free tip separation is present in $\mathrm{R} 1$ at the casing end. There is a saddle point at the leading edge of the rotor blade at $70 \%$ span. A separation surface is stretched from this point and extends across the whole passage from suction to pressure side. As the flow enters this condition there would be a discontinuity in the topology of the flow. The saddle point must be created at the leading edge when the flow jumps from the attached branch of solutions to the separated branch of solutions. Cell behaviour is observed in an unsteady calculation modelling a third of the annulus. The cells occupy 2 rotor pitches, the flow downstream of R1 is plotted in Figure 17.

Figure 18 shows the equilibrium topology predicted on the working line. At this operating point an open corner separation is present in $\mathrm{S} 1$ at the hub end and a single saddle point sits at the leading edge near the fillet. There is also a double open corner separation in R2. This has two saddle points, one at the leading edge near the fillet and one at the leading edge at $30 \%$ span. When an unsteady simulation is performed large cells are observed that extend over both rows. The cells occupy

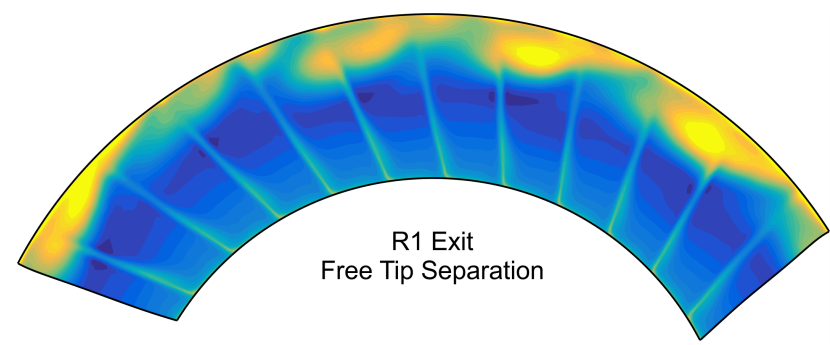

Fig. 17. SNAPSHOT OF CELL BEHAVIOUR AT R1 EXIT 


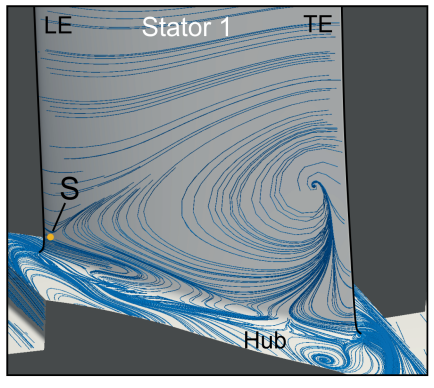

Open corner separation

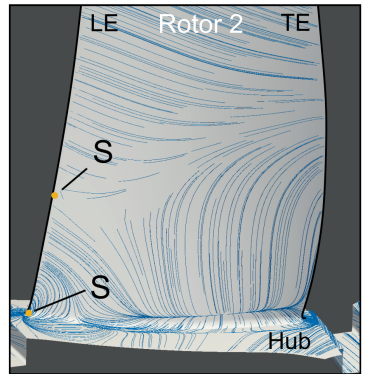

Double open corner separation
Fig. 18. EQUILIBRIUM TOPOLOGY OF SEPARATED FLOW ON DATUM S1 \& R2

approximately 6 stator pitches and 4 rotor pitches and they rotate at $30 \%$ of the shaft speed.

In this part of the paper two operating points have been investigated that have separations with a topological discontinuity, cell behaviour is also observed at both conditions. If the cell behaviour can be removed, unsteady mechanical loading on the compressor can be reduced. This is the focus of the next section of the paper.

\section{Redesign for Axisymmetric Flow}

Previous work has shown how 3D design can be a powerful tool in controlling 3D separation mechanisms. Taylor \& Miller [3] showed how sweep and lean controls the strength of the transverse pressure gradient (TPG) and therefore the quantity of transverse flow on the blade surface. By increasing the TPG the separation mechanism on a blade with a fixed end can be changed from an open corner separation to a trailing edge separation. Hewkin-Smith et al. in [11] showed how sweep and lean changes the distribution of axial momentum in a tip leakage jet. By increasing the axial momentum of the jet or pushing its distribution rearward it was possible to change the route to tip separation from a tip leakage dominated mechanism to a corner separation dominated mechanism. In this part of the paper these design philosophies will be applied to the problem of R1 free tip separation and S1 \& R2 open corner separations.

In a high-speed rotor design it is difficult to apply lean because of centrifugal loading so further sweep is added to the tip of R1 to improved the free tip separation. Figure 19 shows

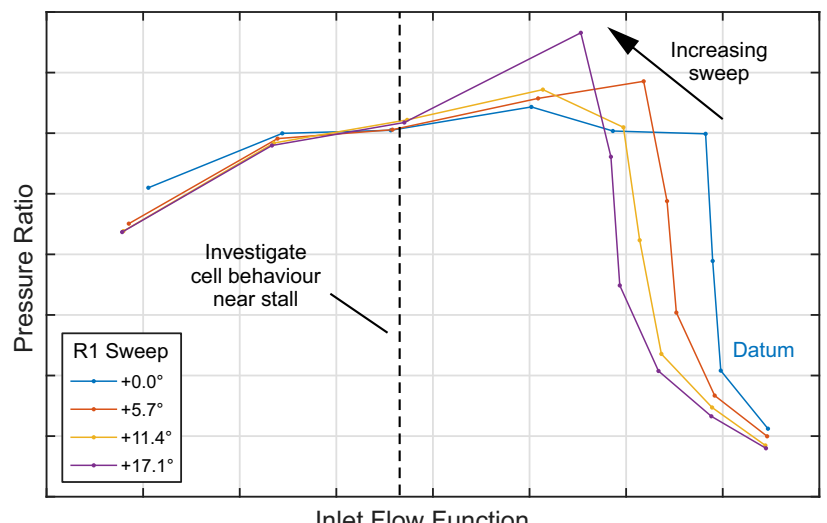

Fig. 19. NEAR STALL CHARACTERISTICS OF R1 DESIGNS
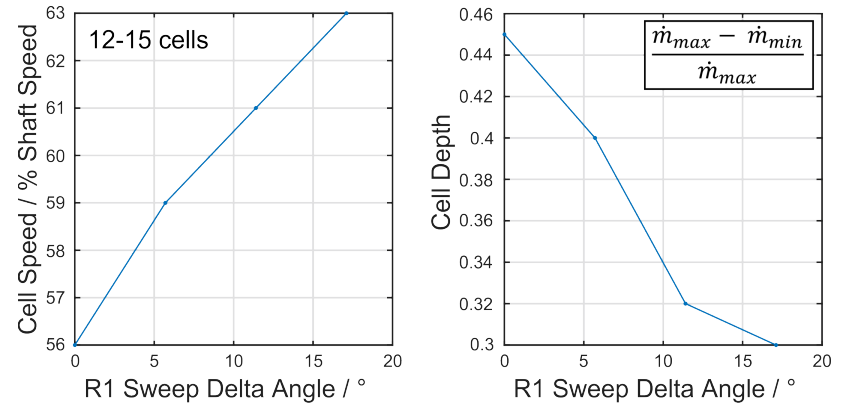

Fig. 20. EFFECT OF INCREASED R1 SWEEP ON CELL BEHAVIOUR

Table 2. INCREASED LEAN \& SWEEP IN S1 \& R2 DESIGNS

\begin{tabular}{lllll} 
3D design factor & $-20 \%$ & $+20 \%$ & $+60 \%$ & $+100 \%$ \\
\hline S1 hub sweep & $-1.4^{\circ}$ & $+1.4^{\circ}$ & $+4.2^{\circ}$ & $+7.0^{\circ}$ \\
S1 hub lean & $-3.2^{\circ}$ & $+3.2^{\circ}$ & $+9.5^{\circ}$ & $+15.9^{\circ}$ \\
R2 hub sweep & $-1.0^{\circ}$ & $+1.0^{\circ}$ & $+3.0^{\circ}$ & $+5.0^{\circ}$ \\
R2 hub lean & $-1.2^{\circ}$ & $+1.2^{\circ}$ & $+3.6^{\circ}$ & $+6.0^{\circ}$
\end{tabular}

the characteristics of designs which have up to $17.1^{\circ}$ of increased sweep. It shows that sweep increases the maximum attainable pressure rise and reduces the critical flow rate at which S1 \& $\mathrm{R} 2$ hubs recover.

The flow at an operating point where R1 separations are present is investigated with unsteady simulations performed on a third of the annulus. The effect of sweep on the cell behaviour in $\mathrm{R} 1$ is presented in Figure 20. By increasing the sweep by $17.1^{\circ}$ the speed of the cells is increased by $13 \%$. The depth is measured by the minimum mass flow in any passage and is reduced by $33 \%$ with increased sweep. However, 12-15 cells are present in all designs and the changes to speed and cell depth are relatively modest for the large quantity of sweep that is applied.

This is because the 3D design has not changed the topology of the separated flow. Hewkin-Smith et al. showed that 3D design could change the route to tip separation in [11] but it cannot change the topology of the separation once it has occurred. Large discontinuities are still present in all of the designs and so cell behaviour is always observed.

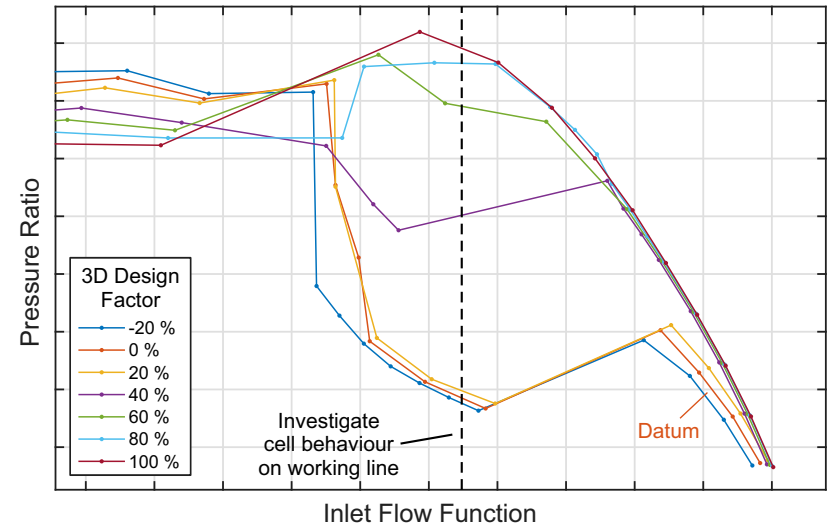

Fig. 21. NEAR WORKING LINE CHARACTERISTICS OF S1 \& R2 DESIGNS 


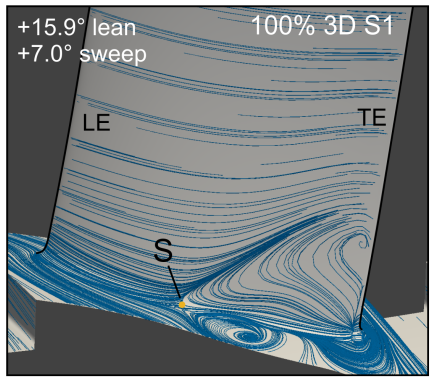

Small open corner separation

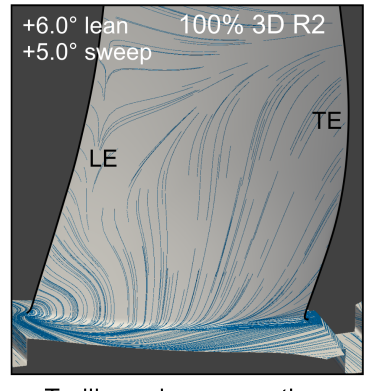

Trailing edge separation
Fig. 22. EQUILIBRIUM TOPOLOGY OF SEPARATED FLOW ON 3D REDESIGN OF S1 \& R2
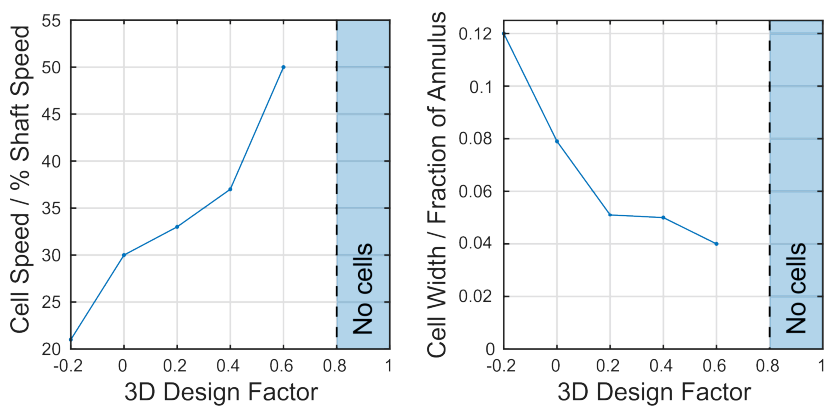

Fig. 23. EFFECT OF S1 \& R2 3D DESIGN ON CELL BEHAVIOUR

3D design is applied to $\mathrm{S} 1 \& \mathrm{R} 2$ to attempt to reduce the cell behaviour that occurs on the working line. Sweep and lean is applied to both blade rows in a fixed ratio, thus it is possible to describe the magnitude of added 3D design with a single parameter, the 3D design factor. Examples of the lean and sweep added to S1 and R2 hubs are described in Table 2. Note that the datum design is at $0 \%$ and the maximum quantity applied is at $100 \%$. The effect of these design changes on the characteristics is shown in Figure 21. Close to the working line the 3D redesign has a dramatic effect on the shape of the characteristics. The collapse in pressure rise caused by the open corner separations can be removed completely.

The equilibrium topology as predicted in steady simulations is shown for the 3D redesign in Figure 22 this should be compared with the datum separated topologies in Figure 18. In S1 there is still an open corner separation, however its size is reduced. The saddle point sits at $50 \%$ chord instead of at the leading edge, which means that the magnitude of the topological discontinuity is also reduced. In R2 there is now a large trailing edge separation instead of a double open corner separation, so the topology will change smoothly to arrive at this operating condition.

The effect of the redesign on the predicted cell behaviour is shown in Figure 23. 3D design has a powerful effect on cells which are located on the fixed ends of blade rows. In this case the speed of the cells can be increased from $20 \%$ of the shaft speed to $50 \%$ or more. The width of the cells measured by the fraction of annulus they extend over is reduced from $12 \%$ down to $4 \%$. The depth of the cells is also reduced and so the unsteady forcing on the blades and shaft will be lower. In the cases of $80 \%$ and $100 \%$ 3D design no cell behaviour is observed. Now the flow is axisymmetric over the whole operating range until R1 separates.
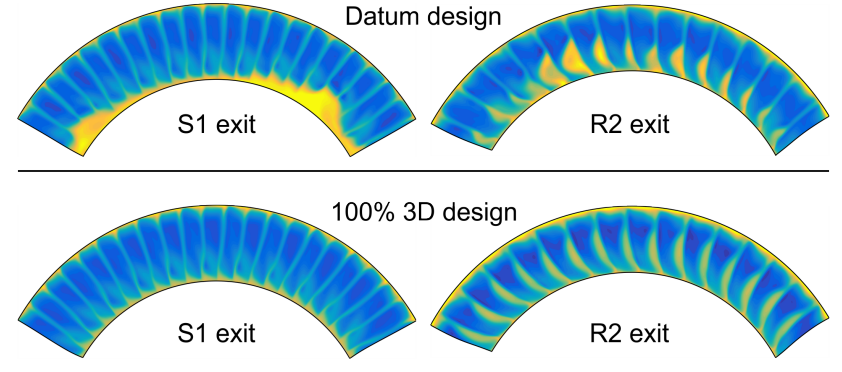

Fig. 24. SNAPSHOT OF CELL BEHAVIOUR IN REDESIGNED S1 \& R2 RUNNING ON WORKING LINE

This can be seen in the contour plots shown in Figure 24, which shows axisymmetric separations in both $\mathrm{S} 1$ and $\mathrm{R} 2$.

This part of the paper has shown how 3D design is extremely powerful in changing separation class in the fixed ends of blades. However, it has little effect on the free tip separation, such as that seen in R1. In this paper relatively large quantities of sweep and lean were required to achieve axisymmetric design. Although this may not always be possible in practice, a designer should always aim to reduce the size of topological discontinuities and achieve a more favourable separated behaviour at off design conditions. This can be achieved by inspecting the topological changes of steady simulations as they are throttled to off-design conditions. If it is not possible achieve a design to separate smoothly then non-axisymmetric cell behaviour will always result.

\section{Conclusions}

This paper has shown that discontinuities in the topology of axisymmetric equilibrium solutions of a flow will result in rotating cells of separations in the annulus of a real multi-passage compressor.

Two examples of separation mechanisms were presented in 2D flow. Leading edge separations, which are common to thin aerofoils, have a discontinuous switch in topology. When multiple aerofoils are simulated in a compressor row, cells of single separations are observed to rotate from one blade to another. Trailing edge separations, which are common to thick aerofoils, have smooth changes in topology and therefore always behave axisymmetrically.

Four examples of separation mechanisms were presented in 3D flow: Midspan leading edge separations occurring on high aspect ratio blades with thin sections have a discontinuity in topology. Trailing edge separations occurring on thick blades with large quantities of sweep or lean have smooth changes in topology; this is the only separation in 3D that does behave smoothly. Both types of endwall separation have discontinuities. In the case of a fixed endwall, corner separations jump from a closed to an open topology. In the case of a free endwall, as at a rotor tip, a leading edge separation is a jump in topology.

3D design can have a powerful effect on the separation type which occurs in a given compressor blade row. It is shown that it is possible to change a compressor which has cells of open corner separations to a design which has no cell behaviour and separates axisymmetrically. The case of separation on a rotor blade tip is more difficult to affect with 3D design, it seems as 
though a discontinuity in topology is unavoidable. While it is not possible to remove the cells it is possible to change their rotation speed and depth.

The aim for the designer is to prevent or reduce the size of discontinuities in the topological response of the compressor blade rows they create. It is possible to assess this by inspecting the topology of steady single passage simulations. If the axisymmetric equilibrium solutions do not respond smoothly then cell behaviour will inevitably result.

\section{Acknowledgements}

The author would like to thank Rolls-Royce plc for their contributions and permission to publish this work. Particular thanks go to Nick Cumpsty who originally started the author's work on this problem. John Dodds, Chris Hall, Ivor Day, Chris Freeman and Ed Greitzer for their crucial advice and discussions. Rob Miller, Tony Dickens and Simon Gallimore for references and perspective. TURBOSTREAM for the use of their solver

\section{References}

[1] Dodds, J., and Vahdati, M., 2015. "Rotating stall observations in a high speed compressor part i: Experimental study". Journal of Turbomachinery, 137(5), p. 051002.

[2] Dodds, J., and Vahdati, M., 2015. "Rotating stall observations in a high speed compressor part ii: Numerical study”. Journal of Turbomachinery, 137(5), p. 051003.

[3] Taylor, J. V., and Miller, R. J., 2016. "Competing threedimensional mechanisms in compressor flows". Journal of Turbomachinery, 139(2), Oct., pp. 021009-021009-10.

[4] Brandvik, T., and Pullan, G., 2010. "An accelerated 3D Navier Stokes solver for flows in turbomachines". ASME J. Turbomach., 133(2), Oct., pp. 021025-021025.

[5] Spalart, P., and Allmaras, S., 1992. "A one equation turbulence model for aerodynamic flows.”. AIAA Journal, 94-439, pp. -.

[6] NUMECA, I., and Manual, A., 2000. Numeca international.

[7] Gallimore, S. J., Bolger, J. J., Cumpsty, N. A., Taylor, M. J., Wright, P. I., and Place, J. M. M., 2002. "The use of sweep and dihedral in multistage axial flow compressor blading part 1: University research and methods development". ASME J. Turbomach., 124(4), Nov., pp. 521-532.

[8] McCullough, G. B., and Gault, D. E., 1951. “'examples of three representative types of airfoil-section stall at low-speed,' naca tn 2502, sept. 1951".

[9] Délery, J., 2013. Three-dimensional Separated Flow Topology. ISTE Ltd and John Wiley \& Sons Inc., London, SW19 4EU and Hoboken, NJ 0703.

[10] Gbadebo, S. A., Cumpsty, N. A., and Hynes, T. P., 2005. "Three-dimensional separations in axial compressors". ASME J. Turbomach., 127(2), May, pp. 331-339.

[11] Hewkin-Smith, M., Pullan, G., Grimshaw, S. D., Greitzer, E. M., and Spakovszky, Z. S., 2019. "The role of tip leakage flow in spike-type rotating stall inception". Journal of Turbomachinery, 141(6), Feb., pp. 061010-061010-10. 